\title{
Prenatal findings in a fetus with X-linked recessive type of chondrodysplasia punctata (CDPX1): a case report with novel mutation
}

\author{
Guannan $\mathrm{He}^{1+}$, Yan Yin ${ }^{2+}$, Jing Zhao ${ }^{1 *} \mathbb{D}$, Xueyan Wang ${ }^{2^{*}}$, Jiaxiang Yang ${ }^{1}, \mathrm{Xi}_{\mathrm{C}}$ Chen ${ }^{1}$, Li Ding ${ }^{3}$ and Yan Bai ${ }^{1}$
}

\begin{abstract}
Background: X-linked recessive chondrodysplasia punctate (CDPX1) is a rare congenital disorder of bone and cartilage development, caused by a mutation in the arylsulfatase E (ARSE) gene located on chromosome Xp22.3. Although most of the affected men had mild symptoms, some had more severe symptoms, and had a poor prognosis.

Case presentation: We present the case of a male fetus diagnosed with CDPX1. Ultrasound clearly showed that hypoplasia of the midface, flatness of face, low flatness of the nose, collapse of the tip of the nose, accompanied by severe spinal stenosis and secondary ossification center of the femoral metaphysis appeared in advance. Chromosome analysis of the amniotic fluid cells revealed $46, X Y$. Whole exome sequencing showed that there was a novel missense mutation of c.640G > A in ARSE gene on X chromosome. Three protein function prediction software FATHMM、Polyphen-2、PROVEAN have shown that the novel missense mutation of c.640G > A in this study was pathogenic.

Conclusions: Our case is a novel mutation and presents a typical characterization of the disease, which can expand the spectrum of mutations of the ARSE gene and is helpful for prenatal ultrasound diagnosis of this disease.
\end{abstract}

Keywords: X-linked recessive chondrodysplasia punctate, Arylsulfatase E, Prenatal ultrasound, Whole exome sequencing

\section{Background}

X-linked recessive chondrodysplasia punctate (CDPX1; MIM\#302950) is a rare congenital disorder of bone and cartilage development, caused by a mutation in the arylsulfatase E (ARSE, MIM*300180) gene located on chromosome Xp22.3 [1]. It was first described by Curry et al. [2], and the incidence of CDPX1 is 1:100000 live births [3]. Maternal immune system disease, exposure to alcohol, or drugs was aetiological factor of chondrodysplasia punctate (CDP) [4-6]. CDPX1 is characterized by punctate calcification in areas of

\footnotetext{
* Correspondence: zhaoj76@163.com; wanxueyang@163.com

${ }^{\dagger}$ Guannan He and Yan Yin contributed equally to this work.

${ }^{\dagger}$ Guannan He and Yan Yin were equally contribute to this study and thus shared the co-first authorship.

'Department of Ultrasound, Women and Children's Hospital of Sichuan Province, No.290, Shayan West 2nd Road, Chengdu 610031, Sichuan Province, China

2Department of Prenatal Diagnosis, Women and Children's Hospital of Sichuan Province, No.290, Shayan West 2nd Road, Chengdu 610031, Sichuan Province, China

Full list of author information is available at the end of the article
}

endochondral bone formation, leading to stippled epiphyses, severe nasal and midfacial hypoplasia; ectopic calcifcations often affecting the airways and in particular the tracheal cartilage; and distal phalangeal hypoplasia [7]. However, the condition of patient is only noticed after birth because of facial features or breathing problems. There have been few reports on prenatal diagnosis of CDPX1 by ultrasound examination $[8,9]$. Here we report a fetus with CDPX1, who was affected by a novel ARSE gene mutation.

\section{Case presentation}

A 25-year-old woman, gravida 1 para 0 , was referred to our hospital at 23 weeks of gestation because of ultrasound abnormality of the fetus that abnormal curvature of spine. Pregnant denied history of immune system disease, exposure to alcohol or drugs such as warfarin and phenytoin.

Ultrasound examination with $5-7 \mathrm{M} \mathrm{Hz}$ probe of the fetal system was then performed. Prenatal ultrasound phenotype 


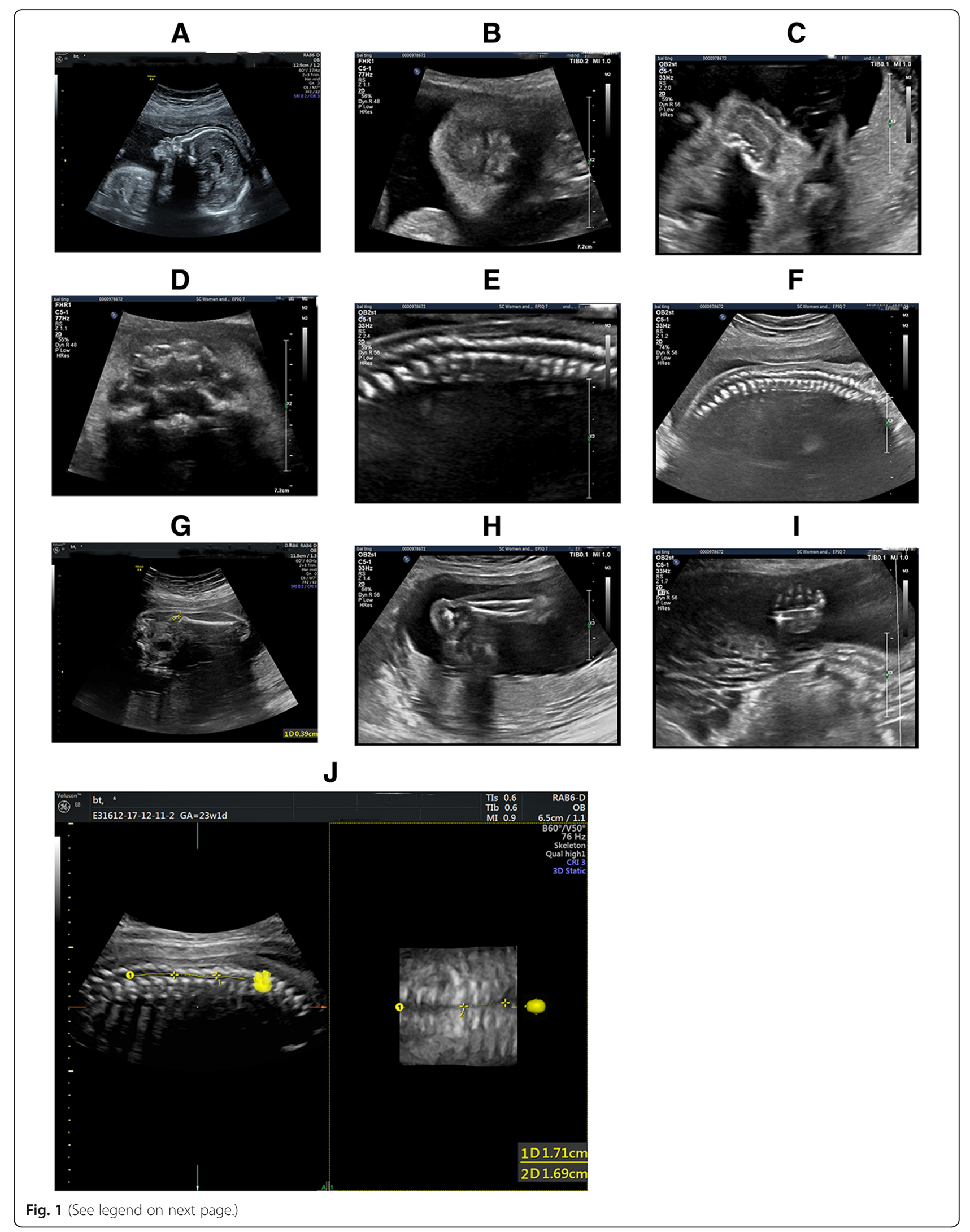


(See figure on previous page.)

Fig. 1 Antenatal ultrasonography features in our patient. a \& b, Sagittal and coronal views of the fetal face showing facial flat, a depressed nose bridge, and collapsed nasal tip. c. Horizontal cross view of the fetal nasal tip show depressed, collapsed, and flat of the nasal tip. $\mathbf{d}$. Transverse view of the upper alveolus shows that the upper alveolus is square. e \& $\mathbf{f}$. Sagittal view of the fetal spine shows changes in physiological curvature, $T_{9}-L_{1}$ spinal canal is narrow, the diameter of the thoracic spinal canal above the stenosis segment was about $4.1 \mathrm{~mm}$, and that of the lumbar segment below the stenosis segment was about $4.0 \mathrm{~mm}$. $\mathbf{j}$. Three-dimensional ultrasound imaging of the fetal spine revealed $T_{9}-L_{1} s p i n a l$ canal is narrow. $\mathbf{g}$. Long axial view of the fetal femur show that early secondary ossification centers. $\mathbf{h} \& \mathbf{i}$. There was no obvious abnormality of fetal ankle joint and hand ossification

are as followed: Fetal external genitals is male, face is flat, nose bridge is depressed, nasal tip is collapse (Fig. 1a-c), and the upper alveolar is square (Fig. 1d). The spine loses normal physiological curvature, some vertebral bodies are small, and the spinal canal is narrow. The T9-L1vertebra is most obvious (Fig. 1e, $f$ and $j$ ). The inner diameter of the narrowest part is only $2 \mathrm{~mm}$. The diameter of the thoracic spinal canal above the stenosis segment was about $4.1 \mathrm{~mm}$, and that of the lumbar segment below the stenosis segment was about $4.0 \mathrm{~mm}$. Secondary ossification centers appear in the epiphyses of both femurs (Fig. 1g, h, i). Based on these ultrasound phenotypes, we recommend that the patient undergo fetal magnetic resonance and amniotic fluid testing to further clarify the diagnosis.

The GE 1.5 T MRI showed the disappeared normal curvature of the fetal spine, partial depression of lower thoracic segment, thinner of muscular layer, and abnormal and narrow down of the spinal canal in the range of about $1.9 \mathrm{~cm}$. Axial view shows abnormal shape of spinal canal and thinning of adjacent spinal cord (Fig. 2).

\section{Genetic analysis}

Chromosome analysis of the amniotic fluid cells was performed with conventional G-banding and revealed 46, XY without any structural aberrations. Parents were informed of the purpose of the study and signed the informed consent. Genomic DNA was isolated from amniotic fluid and peripheral blood leukocytes using the DNeasy Blood \& Tissue Kit (Dusseldorf, Germany) and QIAamp DNA Blood Mini Kit (Dusseldorf, Germany) according to the manufacturer's instructions. Whole exome sequencing: Roche SeqCap EZ MedExome Enrichment Kit was used to perform exome target enrichment. The captured library was sequenced on the illumina Hiseq X-ten Sequencer with PE150 and mean sequencing depth of $100 \times$, and more than $95 \%$ of the area with a coverage of more than 20x. Raw data of exome sequencing in FASTQ format and processed with bioinformatization. A novel missense mutation of ARSE gene c.640G > A (p.G214R) on $\mathrm{X}$ chromosome was found in fetus (refer to the human genome hg19 reference sequence in UCSC database). To further confirm the variant, Sanger sequencing was performed in pedigree. The mother carried the mutation, the maternal grandparents did not have the mutation, the fetus inherited the mutation from the mother (Fig. 3). Three protein function prediction software FATHMM、Polyphen-2、PROVEAN have shown that the novel missense mutation of c.640G > A in this study was pathogenic.

\section{Discussion and conclusions}

CDP is a skeletal disorder characterized by premature ossification of cartilage, which contain different subtypes, including rhizomelic CDP (type1, 2 and 3), CDPX1, X-linked dominant
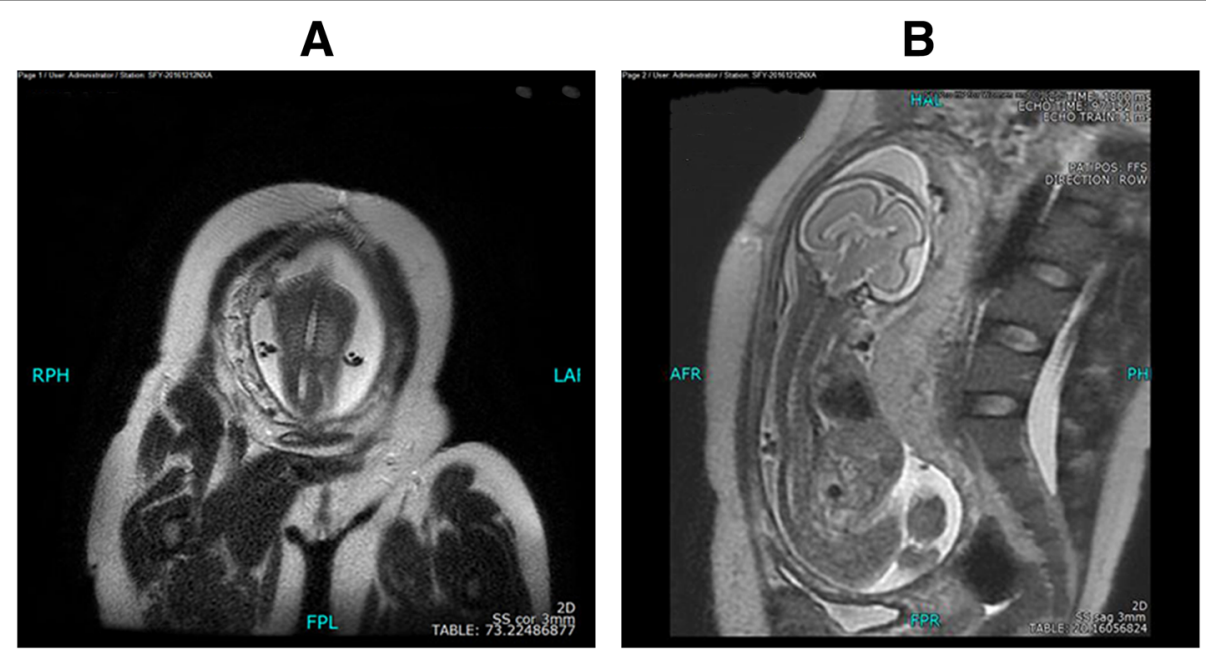

Fig. $2 \mathrm{MRI}$ features in our patient. a \& b. MRI showed the disappeared normal curvature of the fetal spine, partial depression of lower thoracic segment, and abnormal and narrow down of the spinal canal 


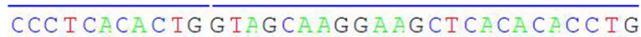

181

193

205

Fetus

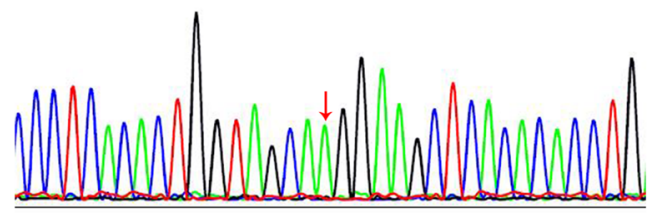

CCCTCACACT G T AGCAAGG AAGCTCACACACCTC

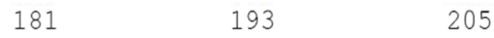

Mother

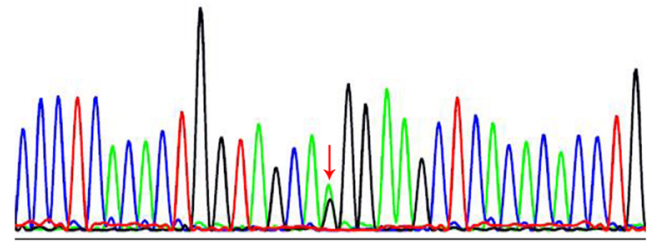

$\overline{C C C I C A C D C T G G T A G C A G G G A G C T C A C A C A C C T G}$

81205

Father

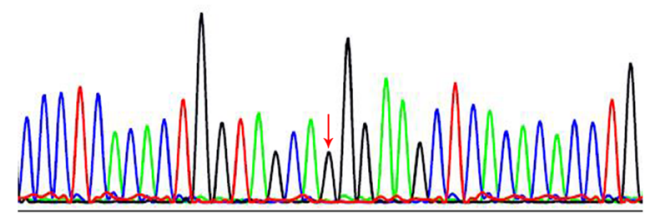

:CCCICACPCTGTAGCAGGGAACTCACACACCTG

81205

Grandmother

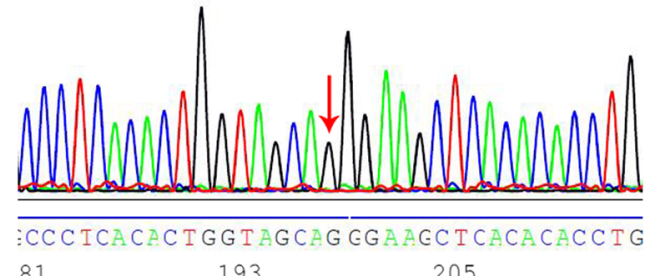

$81 \quad 193$

205

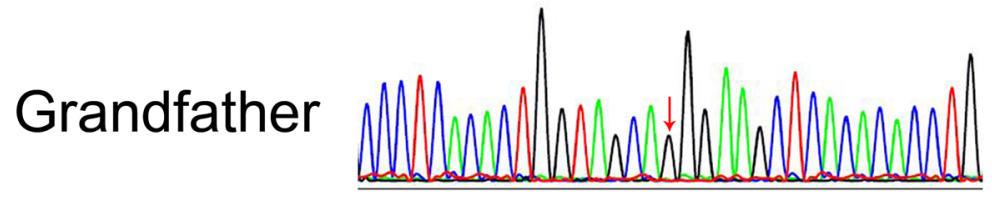

Fig. 3 The sequencing results of the TNSALP gene in pedigree

chondrodysplasia punctate (CDPX2) [10]. CDP is commonly seen in the epiphyses of long bones, the spine, and other uncalcified cartilage, as well as in the trachea and the ends of the ribs.

In previous report, CDPX1 is characterized by brachytelephalangy, punctate calcifications affecting the vertebrae, carpal and metacarpal bones, maxillonasal hypoplasia, and mild developmental delay [11]. Punctate calcification is not a specific feature of the disease and has been found in many diseases, such as Zellweger syndrome, Warfarin embryopathy, Vitamin K reductase deficiency, Trisomy 21, Trisomy 18, and Smith-Lemli-Opitz syndrome etc. [1]. Calcifications can be detected by prenatal ultrasound and $\mathrm{x}$-rays. With the increase of gestational weeks, cartilage begins to ossify and the lesion is often covered by normal calcification, which brings trouble to prenatal diagnosis. Another major abnormality is vertebral involvement, including poor ossification, abnormal shape (wedge, cone, flat), and accompanied by changes in 
the physiological curvature of the spine, which can reflect the degree of vertebral involvement by observing the physiological curvature. The most common facial findings include midface dysplasia, nasal dysplasia and flat cheekbones, similar to prenatal exposure to warfarin in fetuses. Most fetuses are associated with intrauterine growth restriction. There have been few reports on prenatal diagnosis of CDPX1 by ultrasound examination. Symptoms of CDPX1 fetus diagnosed by prenatal ultrasound include nasal hypoplasia, hydramnios, oligohydramnios, long bone dysplasias and vertebrae abnormalities $[8,9,12]$. Although nasal hypoplasia and long bone dysplasias are often considered characteristic changes of Trisomy 21, chromosomal abnormalities can be excluded by amniotic fluid karyotype analysis. Compared with other types, CDPX1 has mild clinical manifestations and good prognosis. Several cases of CDPX1 with disc dislocation or spinal stenosis have been reported with poor prognosis [13, 14].

In our patient, the clinical diagnosis of CDPX1 was based on the ultrasound examination findings with typical facial hypoplasia, vertebrae abnormalities, and premature ossification of the metaphysis of the diaphysis no maternal history of use of warfarin or phenytoin, an inheritance inheritance pattern consistent with X-linked recessive inheritance, and male sex. CDPX1 is caused by a mutation in the gene, which encodes the Golgi enzyme ARSE, antibodies to its enzymes can cross the placenta to the fetus. Genetic analysis revealed that a novel missense mutation of ARSE gene c.640G > A on X chromosome was found in fetus in this report. Further family study revealed that the fetus inherited the mutation from the mother of the novel mutation.

In conclusion, we report a case of a fetus with CDPX1 in whom a novel missense mutation was identified. Our patient was diagnosed by prenatal ultrasound, MRI and genetic analysis. This case is a novel mutation and presents a typical characterization of the disease, which can expand the spectrum of mutations of the ARSE gene and is helpful for prenatal ultrasound diagnosis of this disease.

\section{Abbreviation \\ ARSE: arylsulfatase E; CDP: Chondrodysplasia punctate; CDPX1: X-linked recessive chondrodysplasia punctate; CDPX2: X-linked dominant chondrodysplasia punctate; CMV: cytomegalovirus}

\section{Acknowledgements}

None.

\section{Authors' contributions}

HG and YY conceived and designed this study. WX and Z conducted, analyzed and checked the data. CX and YJ provided materials and samples. BY and DL provided administrative support. All authors read and approved the final manuscript.

\section{Funding}

This study was supported by the Subject of science and technology department of Sichuan Province (No: 18YYJC0624). The funders had no role in the design of the study and collection, analysis, and interpretation of data and in writing the manuscript. The findings and conclusions in this report are those of the author(s) and do not necessarily represent the official position of the funding agencies.
Availability of data and materials

All data generated or analyzed during this study are included in this published article.

Ethics approval and consent to participate

Not applicable.

\section{Consent for publication}

We received written informed consent from parents to publish the information in this case report.

\section{Competing interests}

The authors declare that they have no competing interests.

\section{Author details}

'Department of Ultrasound, Women and Children's Hospital of Sichuan Province, No.290, Shayan West 2nd Road, Chengdu 610031, Sichuan Province, China. ${ }^{2}$ Department of Prenatal Diagnosis, Women and Children's Hospital of Sichuan Province, No.290, Shayan West 2nd Road, Chengdu 610031, Sichuan Province, China. ${ }^{3}$ Department of Radiology, Women and Children's Hospital of Sichuan Province, Chengdu 610031, China.

Received: 17 May 2019 Accepted: 16 July 2019

Published online: 23 July 2019

\section{References}

1. Franco B, Meroni G, Parenti G, Levilliers J, Bernard L, Gebbia M, et al. A cluster of sulfatase genes on Xp22.3: mutations in chondrodysplasia punctata (CDPX) and implications for warfarin embryopathy. Cell. 1995;81(1):15-25.

2. Curry CJ, Magenis RE, Brown M, Lanman JT Jr, Tsai J, O'Lague P, et al. Inherited chondrodysplasia punctata due to a deletion of the terminal short arm of an X chromosome. N Engl J Med. 1984;311(16):1010-5. https://doi. org/10.1056/NEJM198410183111603.

3. Deepthi B, Chhapola V, Kanwal SK, Sharma AG, Kumar V. Chondrodysplasia Punctata with severe airway stenosis. Indian J Crit Care Med. 2018;22(7):5524. https://doi.org/10.4103/ijccm.IJCCM_105_18.

4. Levaillant JM, Moeglin D, Zouiten K, Bucourt M, Burglen L, Soupre V, et al. Binder phenotype: clinical and etiological heterogeneity of the so-called binder maxillonasal dysplasia in prenatally diagnosed cases, and review of the literature. Prenat Diagn. 2009;29(2):140-50. https://doi.org/10.1002/pd.2167.

5. Alrukban H, Chitayat D. Fetal chondrodysplasia punctata associated with maternal autoimmune diseases: a review. Appl Clin Genet. 2018;11:31-44. https://doi.org/10.2147/TACG.S150982.

6. Wester U, Brandberg G, Larsson M, Lonnerholm T, Anneren G. Chondrodysplasia punctata (CDP) with features of the tibia-metacarpal type and maternal phenytoin treatment during pregnancy. Prenat Diagn. 2002; 22(8):663-8. https://doi.org/10.1002/pd.352.

7. Sheffield L, Danks DM, Mayne V, Hutchinson AL. Chondrodysplasia punctata23 cases of a mild and relatively common variety. J Pediatr. 1976;89(6):916-23.

8. Horikoshi T, Kikuchi A, Tamaru S, Ono K, Kita M, Takagi K, et al. Prenatal findings in a fetus with contiguous gene syndrome caused by deletion of Xp22.3 that includes locus for X-linked recessive type of chondrodysplasia punctata (CDPX1). J Obstet Gynaecol Res. 2010;36(3):671-5. https://doi.org/1 0.1111/j.1447-0756.2010.01193.x.

9. Bick DP, Schorderet DF, Price PA, Campbell L, Huff RW, Shapiro L, et al. Prenatal diagnosis and investigation of a fetus with chondrodysplasia punctata, ichthyosis, and Kallmann syndrome due to an Xp deletion. Prenat Diagn. 1992;12(1):19-29.

10. Brunetti-Pierri N, Andreucci MV, Tuzzi R, Vega GR, Gray G, McKeown C, et al. $X$-linked recessive chondrodysplasia punctata: spectrum of arylsulfatase $E$ gene mutations and expanded clinical variability. Am J Med Genet A. 2003; 117A;(2):164-8. https://doi.org/10.1002/ajmg.a.10950.

11. Jeon GW, Kwon MJ, Lee SJ, Sin JB, Ki CS. Clinical and genetic analysis of a Korean patient with X-linked chondrodysplasia punctata: identification of a novel splicing mutation in the ARSE gene. Ann Clin Lab Sci. 2013;43(1):70-5.

12. Garnier A, Dauger S, Eurin D, Parisi I, Parenti G, Garel C, et al. Brachytelephalangic chondrodysplasia punctata with severe spinal cord compression: report of four new cases. Eur J Pediatr. 2007;166(4):327-31. https://doi.org/10.1007/s00431-006-0239-4.

13. Nino M, Matos-Miranda C, Maeda M, Chen L, Allanson J, Armour C, et al. Clinical and molecular analysis of arylsulfatase $\mathrm{E}$ in patients with 
brachytelephalangic chondrodysplasia punctata. Am J Med Genet A. 2008; 146A(8):997-1008. https://doi.org/10.1002/ajmg.a.32159.

14. Herman TE, Lee BC, McAlister WH. Brachytelephalangic chondrodysplasia punctata with marked cenvical stenosis and cord compression: report of two cases. Pediatr Radiol. 2002;32(6):452-6. https://doi.org/10.1007/s00247-001-0638-7.

\section{Publisher's Note}

Springer Nature remains neutral with regard to jurisdictional claims in published maps and institutional affiliations.

Ready to submit your research? Choose BMC and benefit from:

- fast, convenient online submission

- thorough peer review by experienced researchers in your field

- rapid publication on acceptance

- support for research data, including large and complex data types

- gold Open Access which fosters wider collaboration and increased citations

- maximum visibility for your research: over $100 \mathrm{M}$ website views per year

At $\mathrm{BMC}$, research is always in progress. 